\title{
Téoros
}

Revue de recherche en tourisme

\section{Intervenants, produits et entreprises touristiques en Sagamie}

\section{Jean Désy}

Volume 19, numéro 1, printemps 2000

Pour une culture du tourisme au Saguenay-Lac-Saint-Jean

URI : https://id.erudit.org/iderudit/1071800ar

DOI : https://doi.org/10.7202/1071800ar

Aller au sommaire du numéro

Éditeur(s)

Université du Québec à Montréal

ISSN

0712-8657 (imprimé)

1923-2705 (numérique)

Découvrir la revue

Citer cet article

Désy, J. (2000). Intervenants, produits et entreprises touristiques en Sagamie. Téoros, 19(1), 22-29. https://doi.org/10.7202/1071800ar d'utilisation que vous pouvez consulter en ligne.

https://apropos.erudit.org/fr/usagers/politique-dutilisation/ 


\section{InTERVENANTS, PRODUITS ET ENTREPRISES TOURISTIQUES EN SAgamie}

\section{Jean Désy}

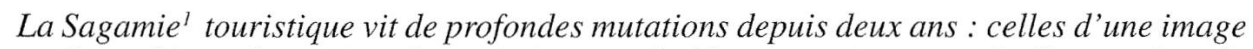
et d'une démarche régionales unitaires, probablement uniques au Québec, et dont la valeur exemplaire pourrait avantageusement être appliquée aux autres régions touristiques du Québec. Voilà le point de départ du présent texte qui tentera de tirer la quintessence de cette ATR (Association touristique régionale) renouvelée et transformée en FTR (Fédération touristique régionale), composée de six ZAT (Zone d'appartenance touristique) et couvrant tout le territoire sagamien. Ce tremplin nous propulsera dans un second temps vers les intervenants, les produits et les entreprises touristiques locales et régionales, dominantes ou en émergence, tant du secteur privé que parapublic au sens large : aperçu quantitatif de la trajectoire empruntée par l'ensemble des acteurs depuis dix ans, suivi d'une analyse détaillée des secteurs du tourisme d'aventure et de l'agrotourisme. Ce circuit récréotouristique se terminera par une douzaine de réalisations issues du monde coopératif, corporatif ou associatif.

\section{UN VIRAGE FÉDÉRATIF PROMETTEUR}

Fort d'une performance touristique évaluée à 1,2 million de journées de séjour et de 152 millions de dollars de dépenses touristiques annuelles en 1995 (Tourisme Québec, 1997), générant quelque 3000 emplois année-personne en Sagamie, le tourisme régional repose néanmoins de façon prédominante sur un marché québécois estimé insuffisant. Il doit « axer davantage ses efforts sur les marchés internationaux » (Tourisme Saguenay-Lac-Saint-Jean, $1998: 5)$ en pleine explosion au cours de la prochaine décennie, maintenir et accrô̂tre sa part de marché, sa visibilité et sa cohérence. Les anciennes structures présentant d'importants problèmes d'équité financière, de liaison et de cohérence interne entre les intervenants locaux, ainsi qu'entre ces derniers et l'ATR, on a donc jeté, en 1997-1998, les bases d'une nouvelle structure cohésive et globale, liée par une charte de partenariat unissant mora- lement les organisations locales intégrées dans des ZAT constitutives de la nouvelle Fédération : liaison morale, mais aussi financière, par un mode de contribution concerté et unifié rejoignant théoriquement toutes les entreprises qui bénéficient des retombées du tourisme (le principe du «profiteur »-payeur).

Couvrant tout le territoire habité de la Sagamie, chacune des six ZAT ${ }^{2}$ est composée de trois types d'organismes qui siègent au conseil d'administration de la FTR : entreprises privées, organismes sans but lucratif et municipalités. Les quelque vingt membres du conseil (dont deux spécialistes en produits d'appel reconnus) mandatent des spécialistes de huit tablesconseil couvrant l'essentiel de la problématique touristique régionale : accueil et information, produits culturels, nature et aventure, développement de la maind'œuvre, commerce et services, hébergement et restauration, festivals et événements et, enfin, commercialisation. Ces tables alimentent la FTR pour qu'elle puisse agir comme « maître d'œuvre de la planification, de l'organisation, de l'orientation, du développement et de la concertation de 1'industrie touristique du Saguenay-Lac-Saint-Jean et conduire toute action propre à animer l'industrie touristique régionale, dont :

- la planification et l'organisation des stratégies d'actions touristiques en région ;

- l'accompagnement et le soutien des six ZAT ;

- la recherche et l'analyse de l'évolution des marchés ;

- la commercialisation extra-régionale » (Association touristique du SaguenayLac-Saint-Jean inc., 1998 : 9).

Un document de travail de la Fédération touristique régionale (1999) précise les intrants de la réorganisation de l'industrie touristique régionale ainsi que les mandats détaillés des organismes membres. Qu'il suffise de conclure sur ce point que, depuis un an déjà, les dépanneurs et les entreprises de taxis, par exemple, contribuent à la FTR s'ils désirent bénéficier de ses services ; en outre, l'organisme doit théoriquement générer annuellement près de $600000 \$$ : la moitié en provenance de l'industrie touristique comme telle, le cinquième des municipalités, près de $8 \%$ de l'industrie apparentée (divertissement, équipements, transport), $9 \%$ des fournisseurs de l'industrie, $3 \%$ des services aux personnes (alimentation, vêtements, transport, marchandises diverses et soins personnels de beauté) ; les Amis de l'industrie fournissent près de $12 \%$ de la masse globale. Au total, on dégage ainsi 186000 \$ 
en nouvelles sources de financement (Tourisme Saguenay-Lac-Saint-Jean, 1998 : 15). Les $600000 \$$ sont affectés comme suit : $50 \%$ à la campagne promotionnelle d'été et $13 \%$ à celle d'hiver ; $17 \%$ au fonds de développement et $20 \%$ aux services fédératifs. Á ce jour, cependant, la somme recueillie plafonne à environ $300000 \$$, ne représentant que $15 \%$ du budget global de la FTR.

\section{0-2000 : UN VIRAGE TOURISTIQUE CONTRÔLÉ}

La structure renouvelée de l'ATR produit régulièrement (tout comme les autres régions du Québec) un outil de promotion touristique régionale fiable et récurrent, d'une facture relativement identique chaque année depuis le début des années 1980. Cette intéressante caractéristique du Guide touristique régional nous permettra donc de planter trois balises temporelles dans cette décennie afin de voir évoluer - en qualité et en quantité - les intervenants, les produits et les entreprises touristiques de la fin des années 1980, du milieu et de la fin de la décennie $(1989,1993-1994$ et 1999-2000) ${ }^{3}$. Nous procéderons en quelque sorte à une analyse de contenu sommaire des trois guides, nous limitant à l'analyse des rubriques Activités et attraits, Hébergement et Publicité, en excluant la description des circuits proposés. Nous suggérons une classification des éléments conforme à la nature de cinq des neuf Tables permanentes de la FTR, soit : Produits culturels, Nature et aventure, Hébergement et restauration, Festivals et événements, Commerces et services liés au tourisme ${ }^{4}$.

Voici ce que nous apprennent les tableaux 1 et 2 : globalement, une surprise de taille nous attend, l'apparition ou la disparition de plusieurs brillantes étoiles du firmament touristique régional, ainsi que la promotion au palmarès touristique d'activités autrefois considérées comme banales et de consommation locale.

\section{NATURE ET AVENTURE}

Ainsi, il y a dix ans, le produit Nature et aventure ne semblait interpeller pour l'essentiel que les Parcs et les zec, mais surtout les pourvoiries avec la pêche et la chasse comme attractions principales. Aujourd' hui, les douze zec ne figurent plus

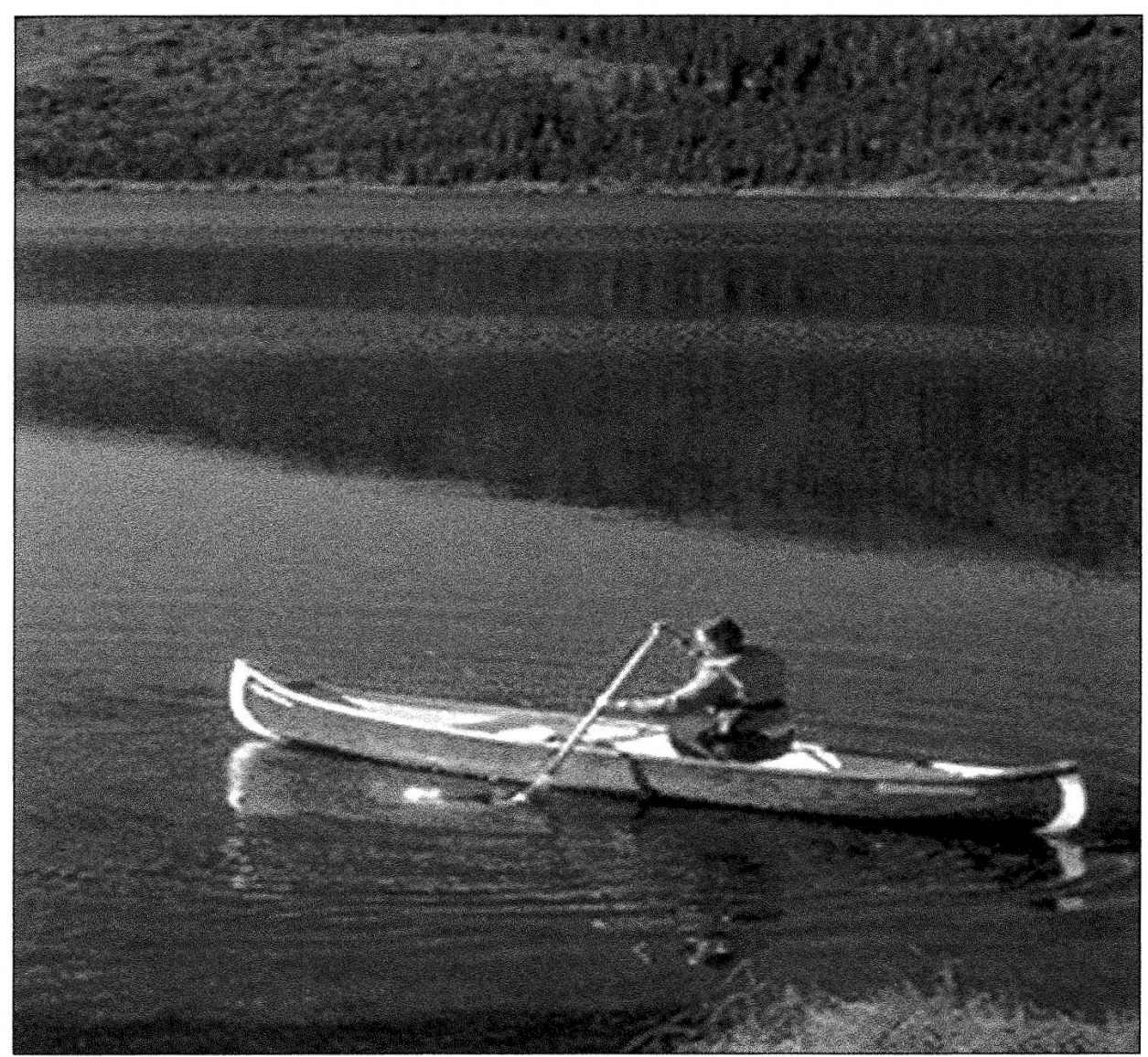

dans les guides depuis longtemps et le nombre de pourvoiries inscrites a chuté dramatiquement (de 45 à 9) ${ }^{5}$. Par contre, les Parcs et les Réserves ont nettement progressé, de pair avec le décollage fulgurant depuis seulement cinq ans du tourisme d'aventure ou de l'écotourisme haut de gamme, avec au moins trois fois plus d'entreprises spécialisées. Détail amusant, le sous-titre de la rubrique Aventure du Guide 1989 : «Espadrilles et sacs à dos », modèle maintenant détrôné par les robustes et coûteuses bottes de randonnée... Le nombre des sentiers de marche a presque doublé en cinq ans (1989-1994) pour se maintenir à 32 actuellement. Grande inconnue dans les deux premiers guides, la motoneige s'impose lourdement dans les 14 clubs listés en 1999. Quant aux entreprises de chiens de traîneau, absentes en 1989, elles ont doublé depuis cinq ans.

Mais le contre-pied de cette forte offensive de l'écotourisme, c'est la faiblesse, voire la décroissance des investissements publicitaires de ce secteur dans le guide depuis dix ans! Petites et nouvelles entreprises, pour la plupart, elles préfèrent sans doute investir dans les dépliants et les sites Internet.

\section{PRODUITS CULTURELS}

Si les Produits culturels ont conservé sur ces dix ans la quinzaine de musées et de haut lieux touristiques reconnus, par contre les théâtres d'été ont totalement déserté les deux derniers guides et les entreprises industrielles ont réduit de moitié le nombre de visites proposées de leur usine. Dans le premier cas, tout se passe comme si la culture du thêâtre d'été avait perdu son attrait et son statut touristiques, « reciblant » plutôt la clientèle régionale ; mais il se peut aussi que le monde théâtral ait misé en bloc sur d'autres véhicules publicitaires comme les journaux locaux et régionaux. Par contre, soulignons que les Produits culturels accaparent presque le quart des espaces publicitaires du guide cette année, une nette remontée depuis dix ans.

\section{HÉBERGEMENT ET RESTAURATION}

On peut aussi s'étonner des chamboulements majeurs au chapitre Hébergement et restauration. En effet, il y a dix ans, le guide ne retenait comme lieux de restauration que les tables champêtres, les gîtes 


\begin{tabular}{|c|c|c|c|c|}
\hline \multicolumn{5}{|c|}{$\begin{array}{l}\text { TABLEAU } 1 \\
\text { Inventaire du nombre d'intervenants/organismes/entreprises } \\
\text { iés dans trois éditions du Guide touristique de la Sagamie par secteurs et branches d'activités }\end{array}$} \\
\hline Secteurs & BRANCHES & Nombre d'entreprises en 1999-2000 & $1993-1994$ & 1989 \\
\hline Produits culturels & $\begin{array}{l}\text { Visites industrielles } \\
\text { Musées } \\
\text { Zoo+Val-Jalbert+Lac-Bouchette } \\
\text { Théâtres d'été }\end{array}$ & $\begin{array}{l}9 \\
10+1 \text { à Tadoussac } \\
3 \\
-\end{array}$ & $\begin{array}{c}5 \\
12 \\
3 \\
-\end{array}$ & $\begin{array}{c}19 \\
11 \\
3 \\
13\end{array}$ \\
\hline Nature et aventure & $\begin{array}{l}\text { Parcs et réserves } \\
\text { Chiens de traîneau } \\
\text { Sentiers de randonnée } \\
\text { Canot, kayak, randonnées, etc. } \\
\text { Clubs de motoneige } \\
\text { Sites de pêche blanche } \\
\text { Érablières } \\
\text { Pourvoiries } \\
\text { Zec }\end{array}$ & $\begin{array}{l}11 \text { (dont } 4 \text { extra-régionaux) } \\
9 \\
32 \\
28 \\
14 \\
4 \\
4 \\
3 \\
-\end{array}$ & $\begin{array}{l}10 \\
4 \\
32 \\
8 \\
- \\
4 \\
5 \\
17 \\
-\end{array}$ & $\begin{array}{l}7 \\
- \\
18 \\
8 \\
- \\
- \\
- \\
45 \\
12\end{array}$ \\
\hline $\begin{array}{l}\text { Hébergement } \\
\text { et restauration }\end{array}$ & $\begin{array}{l}\text { Pourvoiries } \\
\text { Centres de plein air (et de ski de fond) } \\
\text { Restaurants } \\
\text { Hôtels-restaurants } \\
\text { Hôtels et auberges } \\
\text { Couette et café d'Agricotour } \\
\text { Couette et café des Gîtes classifiés du Québec } \\
\text { Campings } \\
\text { Chalets } \\
\text { Auberges de jeunesse } \\
\text { Villages d'accueil pour groupes }\end{array}$ & $\begin{array}{l}9 \text { (dont } 2 \text { extra-régionales) } \\
12 \\
25 \\
41+5 \text { en Charlevoix et Côte-Nord } \\
77+9 \text { en Charlevoix et Côte-Nord } \\
32+2 \text { en Charlevoix et Côte-Nord } \\
67+3 \text { en Charlevoix et Côte-Nord } \\
46+3 \text { en Charlevoix et Côte-Nord } \\
38+4 \text { en Charlevoix et Côte-Nord } \\
- \\
-\end{array}$ & $\begin{array}{l}17 \\
11 \\
39 \\
42 \\
73 \\
7 \\
9 \\
38 \\
23 \\
4 \\
5\end{array}$ & $\begin{array}{l}45 \\
7 \\
- \\
- \\
87 \\
- \\
- \\
41 \\
13 \\
4 \\
-\end{array}$ \\
\hline Festivals et événements & & 46 & 23 & 32 \\
\hline $\begin{array}{l}\text { Commerces et services } \\
\text { liés au tourisme }\end{array}$ & $\begin{array}{l}\text { Clubs nautiques } \\
\text { Location de motoneiges et de VTT } \\
\text { Aires de vélos de montagne } \\
\text { Location de vélos } \\
\text { Golf et mini-putt } \\
\text { Centres d'équitation } \\
\text { Plages } \\
\text { Véloroute des bleuets } \\
\text { Étangs } \\
\text { Bleuetières touristiques } \\
\text { Croisières } \\
\text { Centres de ski alpin } \\
\text { Boutiques d'artisanat/souvenirs }\end{array}$ & $\begin{array}{l}13 \\
11 \\
11 \\
15 \\
9 \\
5 \\
19 \\
1 \\
10 \\
6 \\
10+7 \text { en Charlevoix et Côte-Nord } \\
7 \\
28\end{array}$ & $\begin{array}{l}7 \\
5 \\
- \\
3 \\
3 \\
3 \\
- \\
- \\
- \\
4 \\
12 \\
8 \\
12\end{array}$ & $\begin{array}{l}17 \\
- \\
- \\
- \\
7 \\
7 \\
- \\
- \\
- \\
9 \\
6 \\
- \\
-\end{array}$ \\
\hline
\end{tabular}

Source : Éditions 1999-2000, 1993-1994 et 1989 des Guides, compilation de l'auteur. 


\begin{tabular}{|c|c|c|c|c|c|c|}
\hline \multicolumn{7}{|c|}{$\begin{array}{l}\text { TABLEAU } 2 \\
\text { Jé par } 5 \text { des } 9 \text { Tables-conseil et les ZAT } \\
\text { u Guide touristique de la Sagamie }\end{array}$} \\
\hline \multirow[t]{2}{*}{ Secteurs touristioues } & \multicolumn{2}{|c|}{$1999-2000$} & \multicolumn{2}{|c|}{ 1993-1994 } & \multicolumn{2}{|c|}{1989} \\
\hline & PAGES & $\%$ & PAGES & $\%$ & PAGES & $\%$ \\
\hline Produits culturels & 7,75 & 22,6 & 2,5 & 8,6 & 3,0 & 13 \\
\hline Nature et aventure & 2,0 & 5,8 & 1,0 & 3,4 & 2,0 & 8,7 \\
\hline Hébergement et restauration & 8,0 & 23,4 & 6,0 & 20,7 & 7,0 & 30,4 \\
\hline Festivals et événements & 1,5 & 4,4 & - & - & 3,0 & 13 \\
\hline $\begin{array}{l}\text { Commerces et services } \\
\text { liés au tourisme }\end{array}$ & 1,0 & 2,9 & 3,75 & 12,9 & 2,0 & 8,7 \\
\hline ZAT et organismes membres & 11,75 & 34,3 & 12,75 & 44 & 4,5 & 19,5 \\
\hline Organismes extérieurs & 2,25 & 6,6 & 3,0 & 10,3 & 1,5 & 6,5 \\
\hline Total & 34,5 & 100 & 29,0 & 100 & 23,0 & 100 \\
\hline
\end{tabular}

Source : Éditions 1999-2000, 1993-1994 et 1989 des Guides touristiques de I'ATR, compilation de l'auteur.

et les auberges : point mention de restaurants classiques ni de restaurants d'hôtel, ces entreprises ne se considérant sans doute que marginalement touristiques et ne désirant pas s'affilier à l'ATR. Malgré leur arrivée en force en 1994 et en 1999, elles totalisent beaucoup moins que les gîtes d'Agricotour et les Gîtes classifiés du Québec réunis (une centaine actuellement en dépit de nouvelles réglementations sévères) ; ce sont là des étoiles montantes du tourisme régional depuis cinq ans seulement ${ }^{6}$. Le scénario est similaire, mais atténué, pour les chalets dont le nombre a triplé depuis dix ans. Déclin des auberges de jeunesse, des villages d'accueil pour groupes et des pourvoiries, légère remontée des campings, excellente vitalité des centres de plein air et stabilité relative des hôtels et auberges complètent le tableau du secteur.

Par ailleurs, au plan publicitaire, le secteur Hébergement et restauration se partage la même part du gâteau que le secteur culturel en 1999 (une chute sensible depuis dix ans, de 30 à $23 \%$ ).

\section{COMMERCES ET SERVICES}

Les Commerces et services liés au tourisme connaissent une augmentation foudroyante à quelques exceptions près (les clubs nautiques, les centres d'équitation et les bleuetières touristiques), l'essentiel des gains étant le fait des cinq dernières années. Mais là encore, on se surprend de ne voir apparaître que tout récemment dans le guide un certain nombre de lieux et de services touristiques : location de motoneige, de VTT et de vélos, aires de vélos de montagne, plages et étangs de pêche, voire centres de ski alpin! Les croisières qui avaient déjà bien investi le guide il y a dix ans, réussissent maintenant à tripler le nombre d'entreprises actives (avec l'aide de celles qui opèrent à partir de Tadoussac et des environs). Enfin, la Véloroute des bleuets s'impose désormais comme incontournable dans les prestations touristiques jeannoises et bientôt saguenayennes.

Si le secteur Commerces et services avait occupé $13 \%$ des espaces publicitaires du Guide 1994, il n'arrive plus maintenant qu'à un faible $3 \%$.

\section{FESTIVALS ET ÉVÉNEMENTS}

Dernier secteur recensé, à la fois volatil et permanent, celui des Festivals et événements. Nous laissons à d'autres le soin d'en détailler le menu, surtout estival, nous contentant de noter la très grande fluctuation des inscriptions: passer de $32 \%$ à $23 \%$, puis à $46 \%$, en dix ans soulève une énigme, mais ne retenons que la tendance récente à la hausse qui signifie sans doute la ferme volonté de l'ATR ou des promoteurs de positionner résolument les Festivals et événements dans le courant touristique extérieur et non seulement dans les bassins de clientèles locale et régionale.

Là encore, l'effort publicitaire s'est beaucoup dégonflé en dix ans, atteignant $4 \%$ de la couverture en 1999, après un passage à néant en 1994. Soulignons toutefois la fréquente intégration des Festivals et événements à des pages publicitaires territorialisées. Ces dernières sont essentiellement réservées par les municipalités et maintenant les ZAT de la FTR. Elles monopolisent le tiers des espaces publicitaires en 1999, mais $10 \%$ de moins qu'en 1994, par rapport à seulement $20 \%$ en 1989. Une approche territoriale semble donc se dessiner globalement depuis dix ans, résultat sans doute d'un souci d'économie pour chacun des partenaires, mais aussi d'une nette stratégie de front commun, sinon de réseautage : plusieurs intervenants s'allient au sein d'un même territoire municipal ou intermunicipal pour multiplier les répercussions des attraits et des infrastructures.

Enfin, le guide accepte des commanditaires extra-régionaux qui occupent de 6 à $10 \%$ des espaces publicitaires au cours de la dernière décennie (surtout croisiéristes, hôtels, restaurants, transporteurs et localités de la Côte-Nord, de Tadoussac et des environs) ${ }^{7}$.

\section{QUELQUES PRODUITS ET ENTREPRISES VEDETTES}

On l'a constaté, le tourisme d'aventure en Sagamie, mais aussi dans tout le Québec - vit depuis très peu d'années une ascension foudroyante qui réclame une solide structuration du produit et des producteurs. C'est un diplômé en plein air et pionnier de l'UQAC, François Guillot, qui assume la présidence de l'Association des producteurs en tourisme d'aventure du Québec (APTAQ, 125 membres). Il dirige également depuis quelques années 1'entreprise Québec Hors-circuits, une des pionnières en région des randonnées $n a$ ture sur terre, sur mer et sur neige, ici, au Moyen-Nord comme partout au Québec.

Désireuse de couper court au problème de l'improvisation des pratiques dans l'univers des jeunes entreprises, l'APTAQ prépare un code d'éthique musclé et des normes de sécurité incontournables susceptibles de réduire au minimum les déboires et les accidents fâcheux pour la clientèle. En effet, quand la gamme des activités offertes touche le canot-camping, le kayak de mer, le vélo de montagne, le cyclotourisme, la randonnée pédestre, en passant par les activités hivernales, on 
comprend l'impérieuse nécessité de pallier les risques inhérents à ces pratiques. Par ailleurs, la clientèle du tourisme d'aventure provenant à $50 \%$ d'Europe et d'Amérique du Nord pour des séjours de longue durée, le personnel se doit de livrer des connaissances de tout premier niveau sur les territoires parcourus, tant aux plans biophysique que socioculturel.

Une des pistes prometteuses des entreprises du secteur est la création de partenariats serrés avec les collectivités locales. Celles-ci réalisent les infrastructures de base (pistes de ski de fond, refuges, chalets ou tentes, kiosques d'accueil...) et les entreprises animent et encadrent les activités offertes tout en fournissant l'équi- pement, le matériel et le transport. Ce mutualisme s'inscrit parmi les mesures de revitalisation les plus précieuses pour le monde agro-forestier et cette synergie peut s'accroître encore dans les zones périphériques des parcs, comme en témoigne l'exemple de Rivière-Éternité avec Québec-Hors-Circuits.

On peut également souligner un très fructueux partenariat entre les gestionnaires du Cap-Jaseux, vaste parc municipal près de Chicoutimi sur les bords du Saguenay ${ }^{8}$ et Parcours-Aventures, qui assure l'animation et la fréquentation du site avec sa clientèle, en plus de certaines tâches de développement et d'administration. Courts et longs circuits de kayak de mer, escalade,

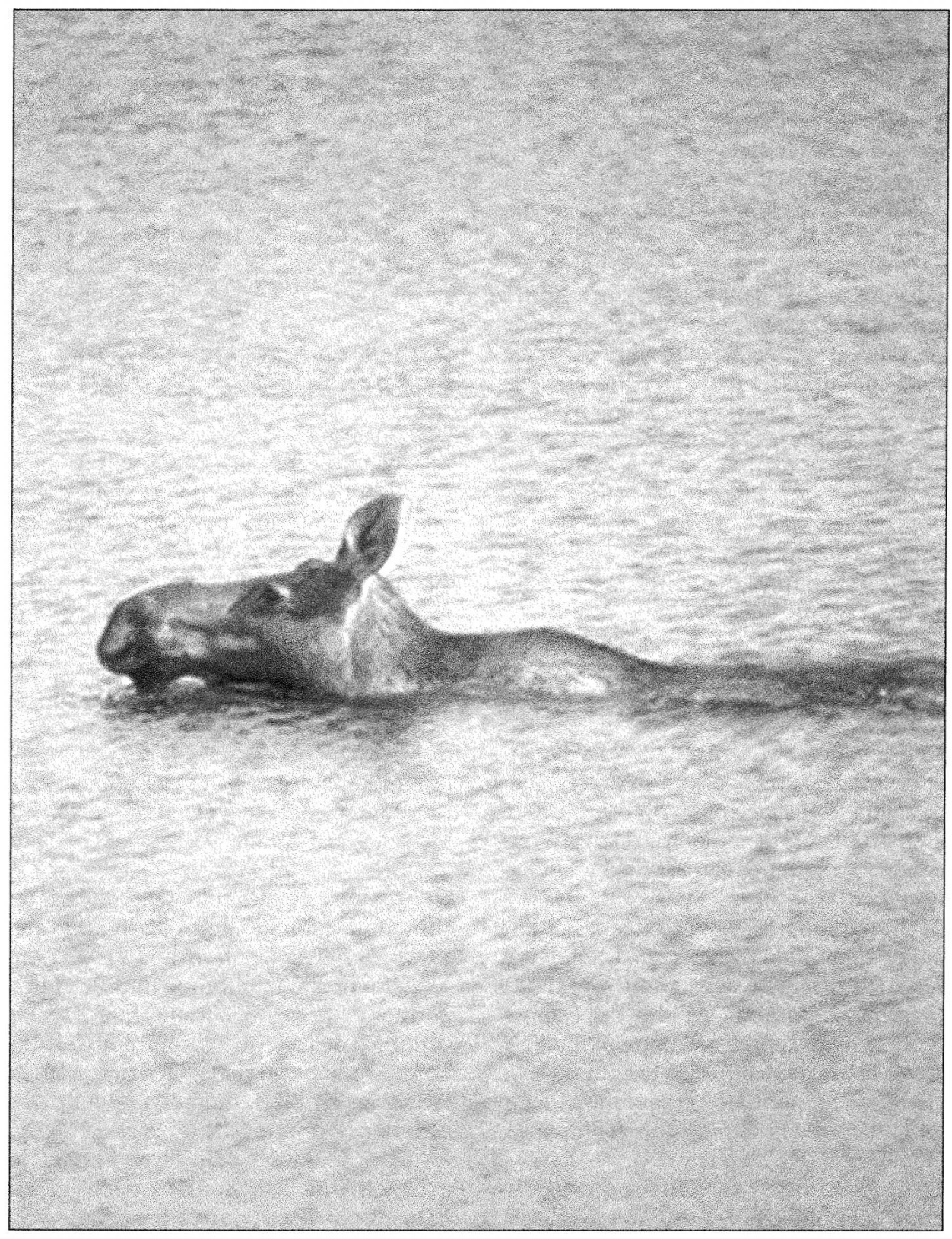

randonnées pédestres et classes nature sont au menu, à partir d'un camp de base, intégré au parc, qui irrigue largement dans la région.

Il existe des alliances profitables similaires entre des entreprises et des pourvoyeurs propriétaires ou locataires de territoires forestiers et de bâtiments situés à distance raisonnable des agglomérations urbaines. Là encore, la complémentarité joue, surtout si la pourvoirie possède déjà son agence de voyage européenne ouverte au tourisme mondial, comme c'est le cas de la Pourvoirie Cap-au-Leste qui s'est dernièrement associée à Québec Hors-Circuits.

L'agrotourisme, comme nous 1'avons souligné, connaît également un essor sans précédent. C'est particulièrement vrai au chapitre de l'hébergement, bien qu'il ne faille pas assimiler chaque gîte B\&B (ou Couette et café) à une entreprise rurale, en particulier les hôtes des Gîtes classifiés du Québec ; c'est bien sûr davantage le cas des gîtes affiliés à Agricotour. Par contre, cet engouement s'appuie sur un éventail impressionnant de productions du terroir, comme en témoigne la deuxième édition d'un récent guide publié dans cette veine (Collectif, 1999-2000) ${ }^{9}$. On y propose en effet neuf thématiques d'appel, certaines traditionnelles, d'autres nettement exotiques : L'hébergement à la ferme, À la découverte de la ferme, Au pays des bleuets, La cueillette de vos fruits et légumes, Des animaux d'ici et d'ailleurs, Les délices de chez nous, Paradis de chasse et pêche, Les petits kiosques à la ferme et $\grave{A}$ dos de cheval.

Si l'on ne propose qu'une douzaine de fermes d'hébergement, elles offrent par contre collectivement beaucoup plus qu'une chambre et un petit déjeuner : table d'hôte de gibiers, chasse au faisan et à la perdrix, visite et vente des produits et des animaux de la ferme, participation aux travaux de la ferme, camping sauvage et activités de plein air, location de chalets, cueillette de bleuets; en outre, elles ont souvent de plus grandes capacités d'accueil que les simples B\&B urbains. Une demidouzaine de fermes et de serres se spécialisent dans les visites guidées ou les visites pédagogiques pour les groupes. Les bleuetières proposées - on en compte dix, plutôt que les six inscrites dans le Guide de l'ATR - , offrent toutes la cueillette et, dans certains cas, des aires de pique-nique, des dégustations et des repas champêtres, 
des visites guidées sur réservation, la vente d'artisanat et les produits de la bleuetière.

Une quinzaine de producteurs offrent la cueillette de fruits et légumes de saison, certains y rajoutant même la vente de fleurs vivaces et annuelles (horticulteurs), d'arbres et d'arbustes (pépiniéristes). Fraises, framboises, carottes, concombres et laitues dominent les productions des champs en matière de cueillette, tandis que les légumes produits en serre sont vendus aux consommateurs.

Depuis une dizaine d'années, les fermes de gibier domestique se multiplient de façon étonnante autour des ratites (émeus, autruches et nandous, 7 fermes), des cerfs rouges et des wapitis ( 3 fermes), des bisons ( 1 ferme), mais aussi de divers animaux (moutons, lamas, chèvres angora, cochons). Au total une douzaine d'entreprises sont listées, ce qui n'épuise sans doute pas le nombre réel de ces fermes dont certaines vendent la venaison et les produits dérivés (le bois de velours des cerfs rouges et des wapitis, notamment), et offrent bien sûr des visites guidées.

La vente directe de fromage et de sirop d'érable a inspiré à quatre producteurs régionaux l'organisation de visites guidées et d'animations diverses, y compris des repas de groupe. Quatre éleveurs de cervidés et de poissons offrent une chasse contrôlée (chevreuils, daims, sikas, cerfs de Virginie et cerfs rouges pour les premiers, ouananiche et truite mouchetée en étang pour les seconds). Finalement, c'est environ trois douzaines de fermes - dont plusieurs mentionnées plus haut - qui offrent la vente en kiosque de produits fermiers de toute nature.

\section{CRÉATURES COLLECTIVES RÉCENTES EN RÉCRÉOTOURISME}

Au-delà des initiatives individuelles ou réseautées que nous avons évoquées, des créations collectives d'envergure occupent de plus en plus le champ récréotouristique depuis quelques années : coopératives de solidarité ou de travailleurs, corporations sans but lucratif et paramunicipales tentent de créer la synergie nécessaire à l'éclosion ou au renflouement d'entreprises en difficulté financière. En voici plusieurs exemples tirés de l'actualité des dernières années :

- Centres de ski alpin et coopératives de travailleurs. En faillite déclarée depuis 1994, les trois plus importants centres de ski de la région ont pu se relever grâce à la reprise intégrale des actifs par les travailleurs de ces stations auprès des créanciers bancaires : Le Valinouët, le Mont-Édouard et le Mont-Lac-Vert. Cette approche change de manière radicale l'ancienne façon de faire des employés qui, en un mot, se responsabilisent totalement du destin de leur entreprise, y compris en se sous-payant, en travaillant à des forfaits inter-stations et en visant l'exploitation quatre saisons des domaines alpins par une incontour-

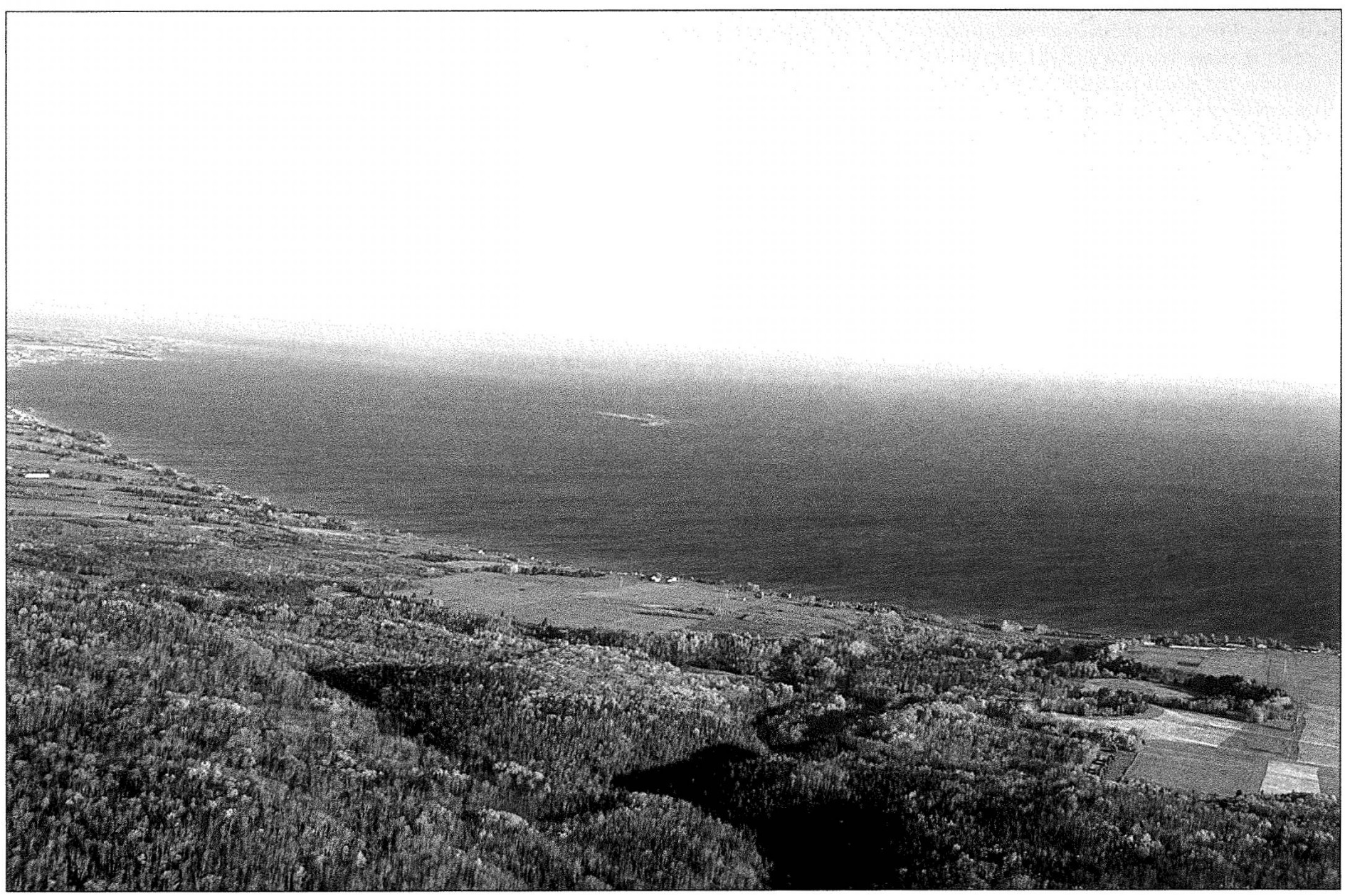

Vue aérienne du Lac Saint-Jean, photo Pierre Lahoud 
nable diversification des prestations et des clientèles (aux plans social et spatial).

- Un projet de coopérative de solidarité à La Doré. Autre entreprise culturelle en manque de 400000 \$ en 1998, Le Moulin des pionniers de La Doré s'est également transformé en une coopérative offrant des parts sociales à divers groupes: travailleurs, utilisateurs, membres de soutien, institutions financières, grandes entreprises, fournisseurs, MRC et paliers gouvernementaux. Les 200 membres espérés devront éponger le déficit et permettre de réaliser un plan d'investissement de quelque trois millions de dollars.

- La SARAS (Société d'aménagement de la rivière aux Sables). Active depuis près d'une vingtaine d'années, ce véhicule paramunicipal jonquiérois a permis non seulement de dégager le lit de ladite rivière de ses millions de «pitounes » submergées, mais de redonner aux citoyens et aux citoyennes la jouissance complète de ce plan d'eau et de ses rives, en plein cœur de la ville : circuit pédestre et cycliste, pêche et pédalo, patinoire sous réflecteurs, place des Nations de la francité, esthétique passerelle d'aluminium reliant les deux rives, le tout agrémenté d'une belle végétation et de boutiques de mieux en mieux adaptées aux lieux. Les fêtes du $150^{\mathrm{e}}$ anniversaire de Jonquière ont permis de compléter ce remarquable parc de la rivière aux Sables qui aura coûté quelque quinze millions de dollars sur vingt ans, financés aux deux tiers par les deux paliers de gouvernement et Hydro-Québec.

- La Corporation d'aménagement de la Petite Décharge. Alma et le Comité ZIP d'Alma-Jonquière ont récemment mis sur pied cet organisme de mise en valeur des rives de cet émissaire du lac SaintJean qui traverse Alma et lui apporte bien des maux issus des rejets de l'activité papetière. Plus modestement que la SARAS, la CAP aménagera quelques kilomètres de sentiers polyvalents, tout en testant quelques scénarios d'utilisation récréotouristique du plan d'eau. Les 14 membres du CA proviennent de multiples secteurs sociaux (municipalités, Alcan et Abitibi-Consol, clubs d'aviron, de chasse et pêche et d'embellissement, en plus de quelques citoyens).
- La Corporation du Circuit cyclable Tour du lac Saint-Jean. Impossible de passer sous silence le tour de force que représente cette «Véloroute des Bleuets », complétée à l'été 1996, maillon majeur de la Route verte, le plus ambitieux projet de réseau cyclable d'Amérique. Ses 256 km de piste ceinturent le lac Saint-Jean et se prolongeront dans quelques années vers Jonquière, Chicoutimi et La Baie, pour rejoindre Tadoussac par Sainte-Rose sur traversier à partir de La Baie (dernier scénario envisagé). Les efforts de cette corporation remontent à 1983, engageant 18 municipalités (y compris Mashteuiatsh), trois MRC et de nombreux partenaires financiers publics et privés. Quelque 2000 cyclistes sont attendus en juin prochain pour en souligner brillamment l'ouverture.

- Les localités de La Baie et de tout le Bas-Saguenay rivalisent d'ingéniosité à ce chapitre et la renommée de ces efforts collectifs a franchi notre oasis prénordique depuis une bonne douzaine d'années. C'est le cas de La Fabuleuse Histoire d'un Royaume notamment, cette fresque historique extravagante qui mobilise chaque année 200 artistes bénévoles six semaines de l'été et qui a réussi à attirer quelque 500000 spectateurs de partout au Québec et ailleurs. On doit aux fêtes du $150^{\mathrm{e}}$ anniversaire de la région la conception et la réalisation de cet événement majeur né de l'homme de théâtre sagamien, Ghislain Bouchard, et régulièrement soutenu par la municipalité. Et c'est au « déluge » de juillet 1996 qu'on doit le projet du monument du millénaire en région, soit la Pyramide des $\mathrm{Ha}$ ! Ha !, « futur joyau culturel et touristique de notre grand Royaume » conçu par l'artiste JeanJules Soucy sur le delta de la rivière $\mathrm{Ha}$ ! Ha! ravagée par le déferlement des eaux soudainement libérées du lac $\mathrm{Ha}$ ! Ha ! tout en amont. Là encore, l'œuvre est vraiment portée par la collectivité.

- Qui par ailleurs n'a réagi au couronnement du roi Denys $1^{\text {er }}$ de l'Anse, dans cette folle aventure de la première monarchie municipale d'Amérique du Nord à l'Anse-Saint-Jean ? Possiblement conçue au départ pour permettre la coûteuse réalisation de la fresque végétale quatre saisons de Saint-Jeandu-Millénaire sur un versant montagneux de la station de ski du MontÉdouard, l'audacieuse monarchie tente ainsi de redresser la courbe d'un trop incertain développement économique local, en mettant notamment en vente des baronnies et des duchés, en plus de tous les attraits locaux. Là encore, les inondations de juillet 1996 ont fouetté les forces créatrices des Anjeannois, ébranlées par la destruction de $20 \%$ des maisons et des infrastructures. Non loin de l'Anse-Saint-Jean, presque toutes les familles de Rivière-Éternité participent avec grande ferveur à l'exposition de crèches, la onzième cette année. On y rivalise d'imagination pour la conception de crèches illuminées de toutes dimensions sur les parterres avant des foyers, tandis qu' on courtise des dizaines d'artisans d'ici et d'outre-mer pour enrichir une exposition de quelque 150 crèches inspirées de cultures régionales et nationales fort différentes. Enfin, le site de la Nouvelle-France de Saint-Félix-d'Otis, récupéré par la municipalité et recyclé du site de tournage de « Robe noire », attire maintenant des touristes chaque saison depuis six ans (de 14000 personnes en 1993 à 28000 en 1999). Ils se laissent guider par Samuel de Champlain à travers les habitations françaises et autochtones de l'époque, voire des fouilles archéologiques actives datant de quatre cents à six mille ans. Cette impressionnante reconstitution d'époque doit le jour à la clairvoyance et à la ténacité du Conseil municipal de Saint-Félix, soucieux de créer une attraction originale et éducative à laquelle ne croyaient vraiment pas les instances politiques nationales, prêtes à détruire les décors en place.

- Abrégeons et terminons ce circuit avec la Pulperie de Chicoutimi - un parc, un site, un musée -, le fleuron du patrimoine industriel régional, sauvé de justesse de la destruction il y a trente ans par la municipalité et le ministère des Affaires culturelles. Haut lieu de la résurgence économique des Canadiens français de la fin du siècle dernier, en lutte contre la domination des Price et compagnie, la Pulperie a connu un recyclage touristique et une énergique restauration en partie compromise et relancée par... le déluge encore une fois ! Ses « vieux » murs abritent donc la maison du peintre naïf Arthur Villeneuve, le musée du Saguenay-LacSaint-Jean, ainsi qu'un parc thématique et des expositions temporaires et permanentes. Les houleux et récents 
débats entre l'actuelle administration municipale et la corporation en place, centrés sur le contrôle, les coûts et le mode de gestion du site, témoignent à tout le moins de la force du débat démocratique qui traverse ce site unique.

\section{FIN \\ DE PISTE...}

Si longue soit-elle, cette analyse des produits, des entreprises et des intervenants touristiques régionaux laisse forcément des vides que plusieurs n'estimeront pas mineurs : la Traversée internationale du lac Saint-Jean, Festirame et le Complexe touristique de la «Dam en terre » à Alma, le Carnaval Souvenir et le parc de la zone portuaire de Chicoutimi par exemple figurent sûrement avec raison sur cette liste prévisible de doléances, en dehors des vedettes consacrées du tourisme jeannois (Val-Jalbert, les sentiers de la nature et l'Ermitage de Lac-Bouchette par exemple). C'est qu'au-delà d'un choix arbitraire de têtes d'affiches régionales, nous avons tenté de mettre en lumière des initiatives peu connues de l'extérieur comme de l'intérieur de la Sagamie, initiatives généralement inscrites dans des tendances récentes sinon lourdes du tourisme régional.

Les entreprises privées émergentes apparaissent pour l'heure d' autant plus petites qu'elles sont nombreuses (agrotourisme et tourisme d'aventure), sans doute vouées à un prochain rééquilibrage de l'offre et de la demande. La taille souvent imposante des entreprises coopératives et corporatives du domaine récréotouristique régional ne surprend guère par la nature même des moyens collectifs mis en œuvre et, quelquefois, en raison de l'énorme poids des dettes qui leur ont donné naissance... Les municipalités ont souvent su drainer la manne gouvernementale par les canaux corporatif ou associatif, mieux adaptés aux critères des organismes subventionnaires. Et on doit reconnaître que nombre d'entre elles ont su encourager la fibre créatrice particulièrement vibrante d'artistes régionaux investis corps et âme dans le développement de leur communauté et même en profiter. Finalement, nombre d'entreprises spectaculaires ont vu le jour grâce à des événements marquants, que ce soit le $150^{\mathrm{e}}$ ou le « déluge » qui, en bout de piste, délient les goussets publics et permettent d'audacieuses réalisations qui drainent d'importants flux touristiques internes et externes. Mais c'est sans doute là le lot de presque toutes les régions...

Jean Désy a un doctorat de troisième cycle en géographie du tourisme; il est géographe en développement local et régional ainsi que professeur associé au département des Sciences humaines de l'UQAC.

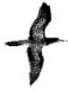

\section{NOTES}

1 Le terme « Sagamie » désigne le SaguenayLac-Saint-Jean (Piékouagami $+\mathrm{e}$ ), régionyme proposé il y a une vingtaine d'années par le géographe Jules Dufour de l'UQAC et dont l'utilisation se répand pour de très nombreuses appellations commerciales et institutionnelles. Il demeure encore officieux et contesté en certains milieux, en particulier par les Jeannois.

2 Il s'agit des ZAT suivantes : Société touristique du fjord, ZAT Jonquière-Plaines-etRives, Initiative touristique Lac-Saint-Jean, Commission touristique Maria-Chapdelaine, ZAT Lac-Saint-Jean Est et Tourisme Chicoutimi-Valin.

3 Les renseignements étaient exacts aux automnes 1988, 1992 et 1998, selon chaque guide.

4 Les trois autres relèvent des processus de fonctionnement interne (Tables des directeurs généraux, de l'Accueil/information touristique, de Commercialisation et de Formation et développement de la main-d'œuvre). L'utilisation de cet outil d'analyse qu'est le Guide annuel doit être interprétée avec beaucoup de circonspection. En effet, n'y sont généralement inscrits que les membres payants de l'ATR, qui peuvent annuler à tout moment leur cotisation annuelle et s'afficher ailleurs... ou pas du tout. Statistiquement peu fiables, ces chiffres veulent plutôt dévoiler

\section{BIBLIOGRAPHIE}

Association touristique du Saguenay-Lac-SaintJean inc. (1998), Charte régionale de partenariat de l'industrie touristique du Saguenay-LacSaint-Jean.

Association touristique régionale et Ministère du Tourisme (1989, 1994-95 et 1998-99), Guide touristique Saguenay-Saint-Jean, 128 p.

Collectif (1999-2000), Le terroir des Bleuets, Guide agrotouristique. Savourez la vie au Saguenay-Lac-Saint-Jean, 22 p. + carte centrale.

Fédération des pourvoyeurs du Québec Inc. des tendances générales de l'évolution des différents secteurs et branches touristiques, qui se sentent ou non solidaires d'un effort promotionnel régional et d'une « industrie » touristique unifiée.

5 Le dernier Guide de la pourvoirie 1999 de la FPQ indique néanmoins la présence de 32 pourvoiries sur le territoire de la région 02 , toutes substantiellement décrites et plusieurs bien annoncées sur 17 pleines pages (environ $8 \%$ des 400 pourvoiries de la FPQ ou des 700 pour tout le Québec). Faut-il en déduire que le médium publicitaire ait migré en grande partie d'un guide à l'autre, d'un premier à base régionale à un second à base sectorielle nationale ? Ce fait nous est confirmé par les gens de la FTR qui signalent, par ailleurs, que certaines pourvoiries se comportent comme les bons vieux clubs privés d'une certaine époque et n'ont que faire de la publicité...

6 Signalons qu'en chiffres absolus la Sagamie se classe dans le peloton de tête des régions québécoises en ce qui a trait au nombre de gîtes touristiques.

7 La FTR considère cependant Tadoussac comme une localité membre qui, à ce titre, ne devrait pas être considérée comme extérieure. De fait, cette station chevauche trois régions touristiques différentes et adhère à trois ATR pour des considérations stratégiques, mais le géographe accepte difficilement de l'intégrer à la Sagamie...

8 Parc qui deviendra peut-être un jour parc régional, selon le vœu des instances administratives qui ont hérité, malgré elles, de ce territoire autrefois sous juridiction d'un OSBL nécessiteux...

9 Ce collectif est le fruit d'une collaboration des quatre organismes suivants : le MAPAQ, le CRDA (Centre de recherche et de développement en agriculture, du Cégep d'Alma), Développement économique Canada et Solution Publicité.
(1999), Guide de la pourvoirie, des vacances grandeur nature, $254 \mathrm{p}$.

Fédération touristique régionale (1999), Réorganisation de l'industrie touristique régionale. Le cadre de mise en cuvre, 19 p.

Tourisme Québec (1997), Le tourisme au Québec en 1995, mars, 102 p.

Tourisme Saguenay-Lac-Saint-Jean (1998), Le développement de notre industrie touristique; un investissement profitable. 